\title{
Fibre-fluorescence immunosensor based on evanescent wave detection
}

\author{
R.G. Eenink, H.E. de Bruijn *, R.P.H. Kooyman and J. Greve \\ Department of Applied Physics, University of Twente, P.O. Box 217, 7500 AE Enschede (The Netherlands)
}

(Received 23rd February 1990)

\begin{abstract}
A fibre-optic sensor capable of detecting an immune reaction between fluorescently labelled antigens and antibodies bound at the waveguide surface is described. In a configuration where skew rays were detected, a signal level was found that made detection of a concentration of $10^{-9} \mathrm{M}$ antigen possible.
\end{abstract}

Keywords: Fibre-optic sensor; Immune reactions

The evanescent wave is a well known physical phenomenon used in several types of chemo-optical sensors such as grating couplers, surface plasmon resonance sensors and fibre-fluorescence sensors [1-4]. It occurs on total internal reflection [5] of plane waves at the interface of two dielectrics and the relatively short penetration depth of the evanescent wave makes it very suitable for measuring optical changes at this interface. Additional specific advantages of a fibre-fluorescence sensor are the long interaction length and the high efficiency for collection of fluorescence emitted near the core of the fibre [6,7]. For these reasons it is attractive to consider the use of the fibre-fluorescence sensor as an immunosensor.

In an evanescent fluorescence immunosensor, an antibody ( $\mathrm{Ab})$, immobilized onto the waveguide surface, is used as a selector molecule to detect quantitatively and specifically the presence of an antigen in the sample to be analysed. This is accomplished by adding a known amount of fluorescently labelled antigen, which will compete for $\mathrm{Ab}$ binding with the antigen present in the analyte solution. On evanescent excitation of the label molecule the resulting fluorescence intensity is a measure of the concentration of non-labelled antigens.

In recent years, several fibre-fluorescence immunosensors with relatively low sensitivity have been described $[2,8-10]$. In this paper it is shown how the sensitivity can be improved considerably by taking into account more properly the propagation of rays inside the fibre with emphasis on skew rays.

\section{THEORY}

Consider a completely decladded fibre with a large diameter relative to the wavelength, so that a geometrical description can be used to determine the best optical set-up. It is of importance to consider how the fluorescent light, excited by the evanescent field, is coupled into the fibre.

Figure 1 illustrates how a ray is propagated inside a cylindrical waveguide. At every reflection the angle $\phi$ between the ray and the normal on the fibre surface remains constant. In general, $\phi$ is greater than the critical angle, $\phi_{\mathrm{c}}$. The angle $\theta_{z}$ 

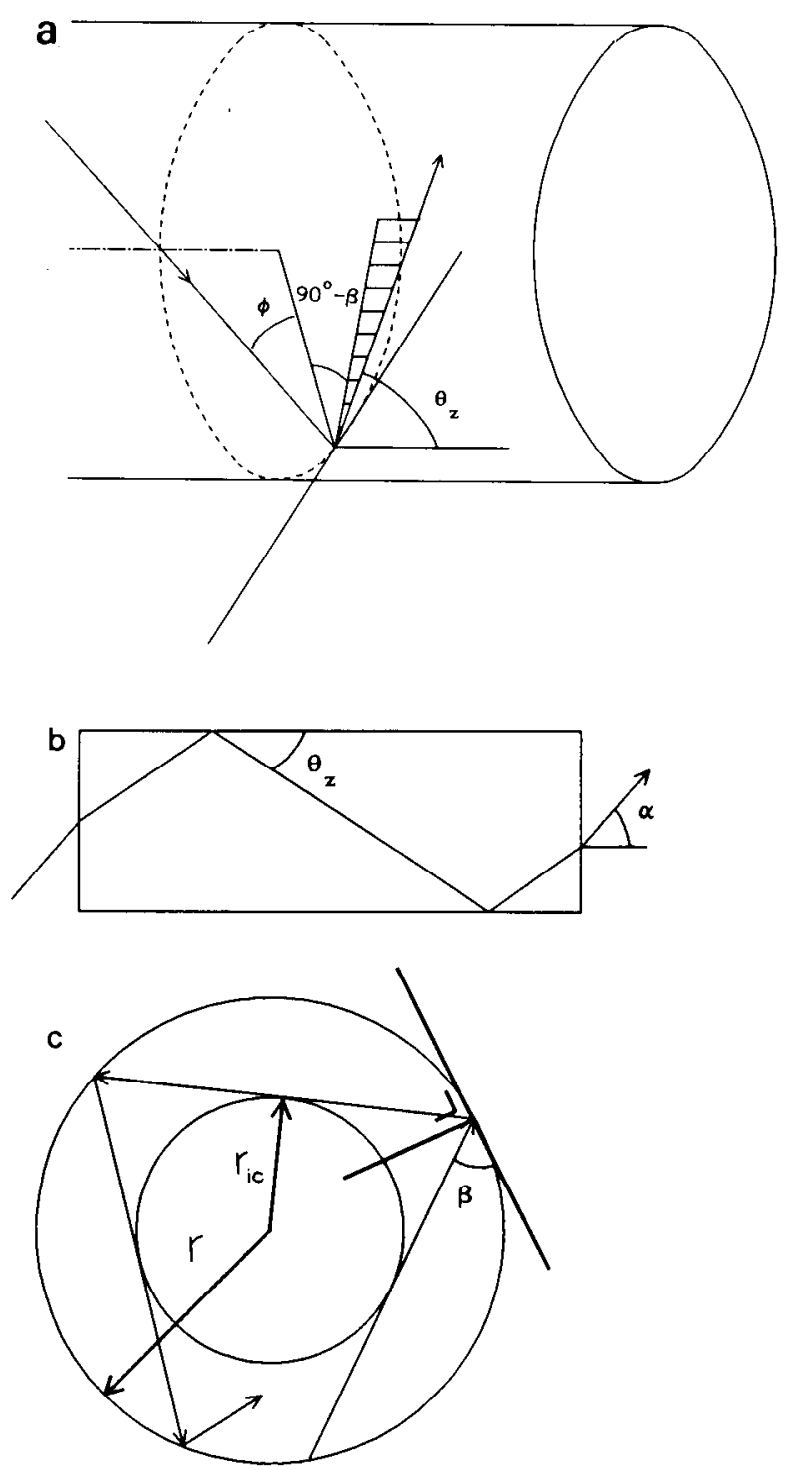

Fig. 1. Geometrical representation of light rays in a cylindrical fibre. (a) The rays are propagated in the fibre when $\phi \geqslant \phi_{c}$, the critical angle. (b) In the special case of $\beta=\pi / 2$, all rays pass through the centre of the fibre and are therefore meridional. (c) when $\beta \neq \pi / 2$ there are skew rays which do not propagate within the inner caustic, $r_{\text {ic }}=r \cos \beta$.

and the azimuthal angle $\beta$ are related by $\phi$ according to [11].

$\cos \phi=\sin \theta_{z} \sin \beta$

The numerical aperture $(N A)$ of the fibre, defined as the sine of the maximum angle $\alpha$ under which light rays can be coupled into the fibre, is then given by

$N A=\sin \alpha=n_{1} \sin \theta_{2, \mathrm{c}}=n_{1} \cos \phi_{\mathrm{c}} / \sin \beta$

where $n_{1}$ is the refractive index of the fibre and $\phi_{\mathrm{c}}=\pi / 2-\theta_{z, \mathrm{c}}$ is the critical angle.

A specific case arises when $\beta=\pi / 2$; then $\phi+$ $\theta_{z}=\pi / 2$ and only meridional rays are present (see Fig. 1b) and

$$
\begin{aligned}
N A_{\text {meridional }} & =\sin \alpha=n_{1} \sin \theta_{z, \mathrm{c}}=n_{1} \cos \phi_{\mathrm{c}} \\
& =\left(n_{1}^{2}-n_{2}^{2}\right)^{1 / 2}
\end{aligned}
$$

where $n_{2}$ is the refractive index outside the fibre.

If $\beta \neq \pi / 2$, then the light is propagated in skew rays (see Fig. 1c). In this instance, according to Fqn. $1, \theta_{z}$ can have values up to $\pi / 2$, and $N A$ increases relative to that for meridional rays and can approach a value of unity (cf., Eqn. 2a).

Various workes $[6,7,12]$ have shown that a radiating dipole, such as a fluorescing label, close to the interface of two dielectrics will radiate its power preferably in directions near the critical angle in the medium with the higher refractive index. In the present case the label molecules are about $5 \mathrm{~nm}$ from the surface. The light rays coupled into the fibre will therefore be concentrated near a cone surface with the symmetry axis normal on the fibre surface and with an internal top angle $2 \phi_{\mathrm{c}}$. A minor part of the rays will have $\theta_{z}=\pi / 2-\phi_{\mathrm{c}}$ and is meridional. Most of the fluorescent power coupled into the fibre will, however, be in the skew rays, which, as can be seen in Fig. $1 \mathrm{c}$, are concentrated near the surface of the fibre. Half of this light will reach the fibre end, and a portion of the light rays with $\theta_{z}$, obeying Eqn. 3, will be captured by the detector.

$\phi_{z, \mathrm{c}} \leqslant \theta_{z} \leqslant \arcsin \left(1 / n_{1}\right)$

This means that the detection of fluorescence at the end of the fibre has to be optimized for detection of the skew rays which are coupled out.

\section{EXPERIMENTAL}

The experimental set-up is shown in Fig. 2. The fibre, with a core diameter of $0.6 \mathrm{~mm}$, was com- 
pletely decladded. The cuvette contents were ca. $250 \mu 1$ and the interaction length of the fibre was ca. $3 \mathrm{~cm}$. A Melles-Griot 543.5-nm helium-neon laser was used, which as a beam diameter of 0.75 $\mathrm{mm}$ and a power of ca. $0.2 \mathrm{~mW}$. The lens used for exciting and collecting the fluorescence was a Spindler \& Hoyer 40/0.65 microscope objective. The light was pointed directly onto this microscope objective. The beam diameter was approximately four times smaller than the lens aperture, giving the possibility of varying the excitation angle, $\alpha$. In these experiments $\alpha$ was not varied because it is difficult to optimize fully the optics of the emission and the excitation paths at the same time. The alignment procedure was stopped if a clear green ring pattern coming out of the fibre was observed.

The magnified image of the fibre end overfilled the entrance pupil of the second lens. Therefore only part of the end surface near the edge was imaged onto the detector because the skew rays due to emitted fluorescence are concentrated there (see Fig. 3). When the centre of the fibre was imaged on the detector the signal was found to be insufficient. Although $N A_{\text {meridional }}$ for the fibre, $\left(1.48^{2}-1.33^{2}\right)^{1 / 2}$, equals $N A$ for the microscope objective and the skew rays are emitted at greater angles, a portion of the skew rays leaving the fibre

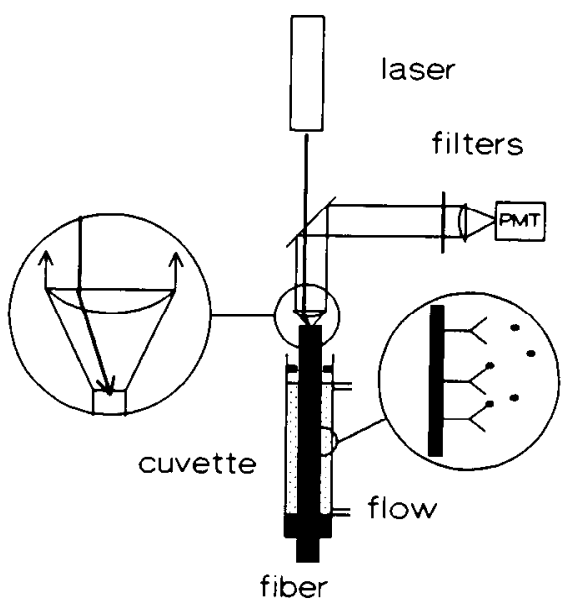

Fig. 2. Experimental set-up used to excitc the fluorescently labelled antigens and detect their emitted light propagating in the fibre. The fibre is held at the bottom of the cuvette only. In the insets the immobilized antibodies and the coupling of light in and out are shown in greater detail.

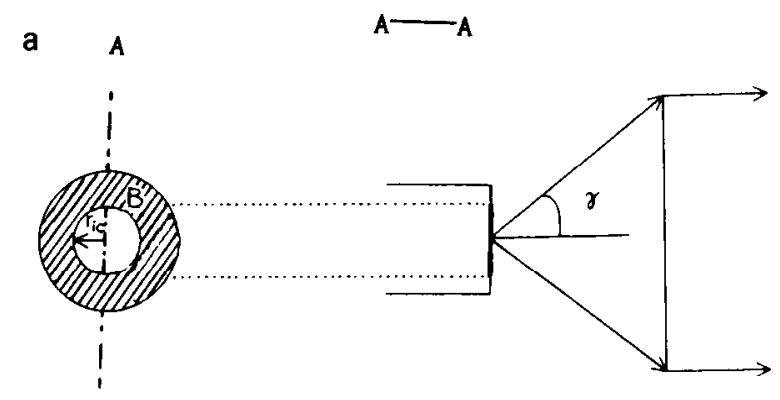

A

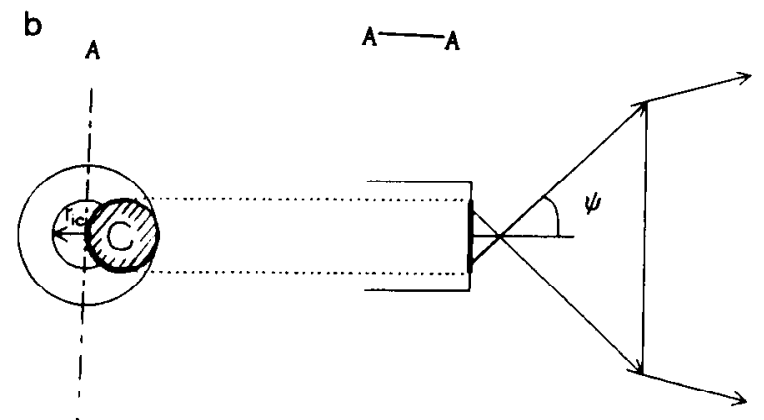

A

Fig. 3. Detection of the fluorescent emission. (a) The skew rays leave the fibre from the ring marked $B$, so ideally this ring should be completely imaged onto the photomultiplier. For detection an objective with $N A=\sin \gamma=0.65$ was used, which allows the detection of all rays from the centre of the fibre with $\gamma \leqslant 40.54^{\circ}$. (b) Part of the skew rays which are emitted from the surface labelled $\mathrm{C}$ at angles $\psi$ greater than $\gamma$ can be detected, however, because they fall on the lens aperture.

end within the area $C$ (see Fig. 3b) could be detected. It is clear that a lens with a greater $N A$ would improve the detection. It has been shown [12] that the choice of a microscope objective with $N A=0.70$ will more than double the signal level compared with the objective with $N A=0.63$ used.

An important fact to consider experimentally is that light rays travelling under the critical angle will be coupled out if the fibre is in contact with a material with $n>1.33$. It is essential that this does not happen before the exciting light reaches the antibody-coated part and before the fluorescence reaches the upper fibre tip. Therefore, the fibre was held only at the far end. The set-up was therefore sensitive to mechanical noise and electronic noise suppression was applied by means of 
phase-sensitive detection using an optical chopper and a lock-in amplifier.

In view of the relatively long characteristic time of the immune reaction, the measuring system time constant could be chosen around $3 \mathrm{~s}$. The antibody anti-human serum albumin (aHSA) was covalently coupled to the fibre surface following a previously described silanization procedure [13]. Subsequently, BSA was added to avoid nonspecific adsorption of antigens at uncovered parts of the surface.

The corresponding antigen HSA was labelled with an average of three molecuels of Rhodamine $B$ isothiocyanate (RBITC) per HSA. RBITC has its absorption maximum near $560 \mathrm{~nm}$ and the emission is at ca. $580 \mathrm{~nm}$. For this dye the use of an optical filter set consisting of three Schott OG570 and one band-pass filter $(579 \mathrm{~nm})$ proved to be adequate. All measurements were performed at room temperature in $0.1 \mathrm{M}$ phosphate buffer (pH 7.15).

\section{RESULTS}

After preparation, the fibres were mounted in the cuvette, which was then filled with phoshate buffer. The background intensity was then measured. Next, HSA-RBITC in phosphate buffer

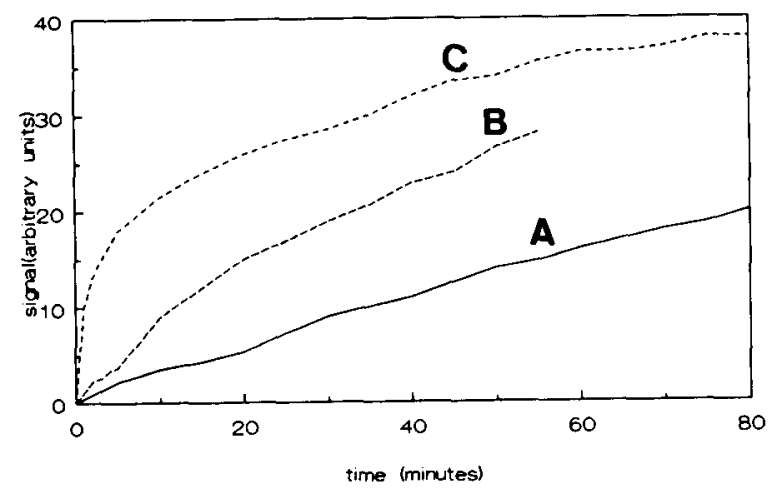

Fig. 4. Immune reactions with (A) $10^{-9}$, (B) $10^{-8}$ and (C) $10^{-7} \mathrm{M}$ HSA-RBITC. For A the relative amplification factor is 80. The measurements for $B$ and $C$ were done without flowing the solution whereas for $A$ the solution was pumped at $4 \mathrm{ml} / \mathrm{h}$ to avoid depletion.

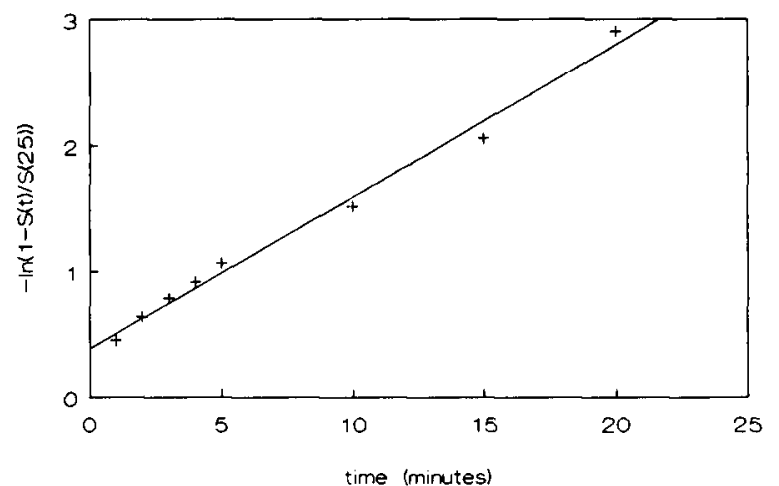

Fig. 5. Curve fit of the immune reaction for $10^{-9} \mathrm{M} \mathrm{HSA-}$ RBITC, giving a time constant of $8.3 \pm 0.3 \mathrm{~min}$.

was added and the immune reaction monitored for ca. $80 \mathrm{~min}$ at room temperature. After the reaction, the cuvette and fibre were rinsed several times but this did not affect the signal level.

An example of three measurements is shown in Fig. 4 for HSA-RBITC concentrations of $10^{-9}$, $10^{-8}$ and $10^{-7} \mathrm{M}$. As is evident, the reaction at the highest concentration is much faster. The curves are reasonably well described by Eqn. 4 which follows from the Langmuir model

$S=\operatorname{constant}[1-\exp (-t / \tau)]$

When Eqn. 4 is fitted to the experimental results, for five independent measurements at $10^{-7} \mathrm{M}$, a $\tau$ (the time constant) value of $10 \pm 2$ min was found. The curve fit for the reaction at $10^{-7} \mathrm{M}$ in Fig. 4 is shown in Fig. 5. The fit is applied up to $t=25$ min as it is clear that after $25 \mathrm{~min}$ the reaction can no longer be described by Eqn. 4. In Fig. 5 it is clear that the fit is valid up to $t=25 \mathrm{~min}$ except for $t=0$. A change from the buffer to the sample takes time and changes the background level, which makes the starting point arbitrary.

For $10^{-8}$ and $10^{-9} \mathrm{M}$ concentrations the reaction did not reach a plateau value, making it very difficult to determine $\tau$. Nevertheless, there is a significant increase in the signal level, indicating that the apparatus is sensitive enough to measure such low antigen concentrations. 


\section{DISCUSSION}

A sensitive fibre-fluorescence immunosensor has been described and its ability to measure the immune reaction of $10^{-9} \mathrm{M}$ HSA-RBITC solutions with good signal-to-noise levels has been demonstrated. The high sensitivity of the sensor could only be achieved because two essential features of the wave propagation were taken into account. First, it was realized that the fluorescence intensity coupled into the fibre is mainly present in skew rays, and the optics used for detection of the fluorescence were optimized for these rays. Second, care was taken to avoid contact of the fibre with material with a refractive index $>1.33$ as this would lead to loss of rays travelling under the critical angle.

The experimental set-up can be improved considerably by using a detection lens with a greater $N A$ and a greater focal length because then a greater portion of the emitted skew rays can be detected. Further improvements could include coating the fibre with a reflecting layer at the position where the fibre is held so that it can also be held at the top and mechanical vibrations are avoided while retaining the light in the fibre. Also, the interaction length could be increased considerably.

In its present form the sensor is already much more sensitive than any fibre-optic fluorecence sensor reported so far, presumably because the two factors mentioned above were not previously taken into account.

The authors thank Ms. Lian Keldermans for the preparation of the fibres and solutions.

\section{REFERENCES}

1 K. Tiefenthaler and W. Lukosz, J. Opt. Soc. Am. B, 6, (1989) 209.

2 K. Newby, W.M. Reichert, J.A. Andrade and R.E. Benner, Appl. Opt., 23 (1984) 1812.

3 R.P.H. Kooyman, H.E. de Bruyn and J. Greve, SPIE 798 Fiber Optic Sensors II, (1987) 290.

4 B. Liedberg, C. Nylander and I. Lundström, Sensors Actuators, 2 (1983) 299.

5 F.J. Milford, J.R. Reitz and R.W. Christie, Foundations of Electromagnetic Theory, Addison-Wesley, Hong Kong. 1979, Chap. 18.

6 C.K. Carniglia, L. Mandel and K.H. Drexhage, J. Opt. Soc. Am., 62 (1972) 479.

7 W. Lukosz and R.E. Kunz, J. Opt. Soc. Am., 69 (1977) 1615 .

8 R.M. Sutherland, C. Dähne, J.F. Place and A.S. Ringrose, Clin. Chem., 30 (1984) 1533.

9 T.B. Hirschfeld and M.J. Block, U.S. Pat., 4447 546, 1984.

10 A.M. Smith, SPIE 798, (1987) 206.

11 A.W. Snyder and J.D. Love, Optical Waveguide Theory, Chapman and Hall, New York, 1983.

12 T.R. Glass, S. Lackie and T. Hirschfeld, Appl. Opt., 26 (1987) 2181.

13 C.F. Mandenius et al., Anal. Biochem., 137 (1984) 106. 